\title{
Zeolite-Assisted Shear Exfoliation of Graphite into Few-Layer Graphene
}

\author{
Gabriela Tubon Usca ${ }^{1,2,3, *}$, Cristian Vacacela Gomez ${ }^{1,4}\left(\mathbb{D}\right.$, Marco Guevara ${ }^{1,5}$, Talia Tene ${ }^{1}$,

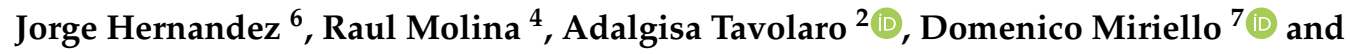 \\ Lorenzo S. Caputi ${ }^{3}$ \\ 1 Physics Research Group, Faculty of Science, Escuela Superior Politécnica de Chimborazo, \\ Riobamba EC060155, Ecuador \\ 2 Research Institute on Membrane Technology (ITM-CNR), Cubo 17C, I-87036 University of Calabria, \\ 87100 Cosenza, Italy \\ 3 Surface Nanoscience Group, Department of Physics, University of Calabria, Via P. Bucci, Cubo 33C, \\ I-87036 Rende, Italy \\ 4 GraphenTech NL, Olympiaweg 28A, 3077AL Rotterdam, The Netherlands \\ 5 Faculty of Mechanical Engineering, Escuela Superior Politécnica de Chimborazo, \\ Riobamba EC060155, Ecuador \\ 6 Faculty of Computing and Electronic, Escuela Superior Politécnica de Chimborazo, \\ Riobamba EC060155, Ecuador \\ 7 Department of Biology, Ecology and Earth Sciences, University of Calabria, Via Pietro Bucci, \\ I-87036 Rende, Italy \\ * Correspondence: gabriela.tubon@espoch.edu.ec
}

Received: 1 July 2019; Accepted: 19 July 2019; Published: 24 July 2019

\begin{abstract}
A novel method is presented to prepare few-layer graphene (FLG) in N-methyl-2-pyrrolidinone (NMP) by using a simple, low-cost and energy-effective shear exfoliation assisted by zeolite and using a cappuccino mixer to produce shear. We propose that the exfoliation of natural graphite flakes can be achieved using inelastic collisions between graphite flakes and zeolite particles in a dynamic colloidal fluid. To confirm the exfoliation of FLG, spectroscopy and morphological studies are carried out using Raman spectroscopy, scanning electron microscopy (SEM) and transmission electron microscopy (TEM). Additionally, the obtained graphene shows a linear flow of current and low resistance. The proposed method shows great promise for the industrial-scale synthesis of high-quality graphene with potential applications in future graphene-based devices, and furthermore, this method can be extended to exfoliate inorganic layered materials such as $\mathrm{BN}$ and $\mathrm{MoS}_{2}$.
\end{abstract}

Keywords: few-layer graphene; zeolite 4A; NMP; mixer

\section{Introduction}

Graphene has generated intense interest due to its two-dimensional structure and its unique set of physical and chemical properties [1,2]. Many research groups have been focused on mapping out and understanding the fundamental properties of graphene [3,4]. In recent years, however, both academic and industrial researchers have been concentrating on fulfilling practical applications for graphene, from high-frequency electronics to smart coatings [5]. Nevertheless, before this can be achieved, it is critically important to develop industrially scalable production methods for graphene and graphene-related materials [6,7]. While graphene can be prepared by several top-down methods (e.g., mechanical exfoliation [8], oxidation-reduction [9-11], liquid-phase exfoliation [12], fast electrochemical exfoliation [13], among others), some important classes of applications, such as conductive coatings and printed electronics, will require access to graphene dispersions or inks. 
Defect-free graphene (of less than five layers) is generally produced by sonicating graphite either in certain solvents [14] or aqueous surfactant [15] solutions. The sonication tends to break up the graphite layers as well as exfoliating them to give large number of graphene nanosheets [16]. Although graphene can be produced by this method, some major problems do appear. The processes involving sonication have limited scalability by the use of extensive sonication time (up to reach high concentrations of dispersed graphene [17]), poor stability of obtained graphene dispersions [18], and high-cost production.

To circumvent these problems, alternative strategies have been proposed such as shear-exfoliation [19] (or turbulence-assisted shear exfoliation [20]) of graphite in stabilizing solvents as well as surfactant solutions. In particular, the shear exfoliation is industrially scalable and employs a rotor-stator mixer to produce graphene in few hours. On the other hand, the addition of surfactants mostly is required in order to stabilize exfoliated graphene due to the surfactant molecules provide the necessary colloidal stability for the graphene dispersion [21]. Nevertheless, the surfactant molecules are electrically insulating and, if they are not removed, could negatively impact the electrical performance of graphene and graphene-based devices [22]. Thus, it is clear that exfoliation techniques to obtain surfactant-free graphene are urgently required.

In this study, we propose an alternative method to prepare graphene dispersions in N-Methyl-2pyrrolidone (NMP) by zeolite-assisted shear exfoliation using a cappuccino mixer to produce shear. NMP is used as dispersant solvent due to its surface tension close to $40 \mathrm{mJm}^{-2}$ [23], which prevents the immediately re-staking and agglomeration of exfoliated flakes. However, NMP is used here to emphasize the role of zeolite instead of the solvent. With this in mind, we propose the use of shear exfoliation assisted by the zeolite $4 \mathrm{~A}(\mathrm{Z} 4 \mathrm{~A})$, in order to improve the exfoliation process and obtain graphene nanosheets. Natural or synthetic zeolites can be used to prepare colloidal suspensions with a low rate of sedimentation [24-27], thus, it is possible to exfoliate graphite for preparing stable and well-dispersed graphene dispersions. Additionally, the obtained graphene shows interesting electrical properties, with potential applications in printed electronics.

\section{Materials and Methods}

Natural graphite flakes were obtained from Pingdu Huadong Graphite Co. Ltd., the NMP (purity 99\%, CAS: 872-50-4) and Z4A (Molecular sieve 4A, powder activated, CAS:70955-01-0) were purchased from Sigma Aldrich. All the chemicals were used as received, without further purification. In this study, we used a cappuccino mixer (Princess 222187 Black, Silver) to disperse and exfoliate graphite. The mixer is composed of a metal wire with $\sim 37$ turns at the end; thus, the speed rotation creates a moderate turbulence. To perform the mix as simply as possible, we added $2 \mathrm{~g}$ of graphite and $2 \mathrm{~g}$ of $\mathrm{Z} 4 \mathrm{~A}$ into $20 \mathrm{~mL}$ of NMP. The mixture was then subjected to controlled shear force using the mixer at $75 \%$ maximum speed rotation $(\sim 10000 \mathrm{rpm})$ for $6 \mathrm{~h}$ in continuous operation. To preserve the intrinsic characteristics of the solvent [28], the exfoliation was achieved at $\sim 26^{\circ} \mathrm{C}$, controlling the temperature every 2 hours. The obtained black suspension was then centrifugated for $10 \mathrm{~min}$ at $1000 \mathrm{rpm}$ to remove non-exfoliated graphite flakes. Then, the supernatant ( $\sim 8 \%$ ) was recovered for the subsequent characterization. For comparison, the experiment was carried out without the presence of Z4A.

The surface morphology and structure of the samples were observed using a field-emission scanning electron microscope (FE-SEM, Quanta Feg 400 F7, FEI) with accelerating voltage of $5 \mathrm{kV}$ and transmission electron microscopy (TEM, JEM-1400) operating at $80 \mathrm{kV}$. Raman spectra were obtained using a Thermo Fisher DXR Raman Microscope with OMNICxi Raman Imaging software 1.0, as well as an incident laser light with a wavelength of $532 \mathrm{~nm}$. The current-voltage (I-V) characteristics of the obtained graphene were measured by a Keithley $2400 \mathrm{C}$ source measure unit (SMU) and I-V curves were plotted in the potential window of $-0.5 \mathrm{~V}$ to $0.5 \mathrm{~V}$. SEM and TEM samples were prepared, from supernatant and precipitate, by drop casting of dispersion onto aluminum substrates and holey carbon grids, respectively. Similarly, Raman samples were prepared on glass substrates. I-V samples were arranged by drop casting of dispersion $(2 \mu \mathrm{L})$ on commercial interdigitated gold electrodes 
(Micrux technologies, ED-IDE3-Au). Five measurement cycles were performed according to avoid self-heating effects.

\section{Results and Discussions}

Several papers have evaluated the process and the yield of exfoliation methods by measuring the concentration of dispersed graphene [12,29]. However, concentration is not an appropriated metric for graphene production because high concentrations only are attainable in low volumes, resulting in small quantities of produced graphene [20]. Hence, we briefly analyze the conventional (tip or bath) ultrasonication, simple shear exfoliation, and turbulence-assisted shear exfoliation, in order to understand the exfoliation mechanism of the proposed method, i.e., zeolite-assisted shear exfoliation (Figure 1).

Ultrasonication uses high-frequency sound waves to cause acoustic cavitation within the fluid. When a cavitation microbubble collapses and bursts in solution induces shock waves on the graphite surface, producing exfoliation of large quantities of defect-free, unoxidized graphene [30,31]. Shear exfoliation uses a high-shear rotor-stator mixer, and very high-local shear rates are realized in the gap between the rotor and stator and in the holes in the stator [19]. This means efficient graphene production is localized close to the rotor-stator. The simple equipment to achieve the turbulence-assisted shear exfoliation is a kitchen blender [20]. The rotating blade mixer produces turbulence which is responsible for the presence of high shear rates that are not localized in any single part of the vessel; the produced high turbulence, therefore, generates shear rates that are high enough to result in exfoliation of graphite.

Since our method, reported in Figure 1, does not produce acoustic cavitation, it could be said that the exfoliation of graphite remains a very hard task. Instead of that, this study demonstrates an effective shear exfoliation of natural graphite flakes into defect-free graphene. Even though the proposed method is not well-defined, we hypothesize that the exfoliation of graphite is produced by a moderate turbulence and the inelastic collisions between graphite flakes and zeolite particles in a dynamic fluid, which will make it more competitive in the scalable production of graphene. Attempts to elucidate the exfoliation mechanism and the yield are currently in progress that involve extending the study to focus on distinct zeolites, low boiling point solvents, and shear-exfoliation time.

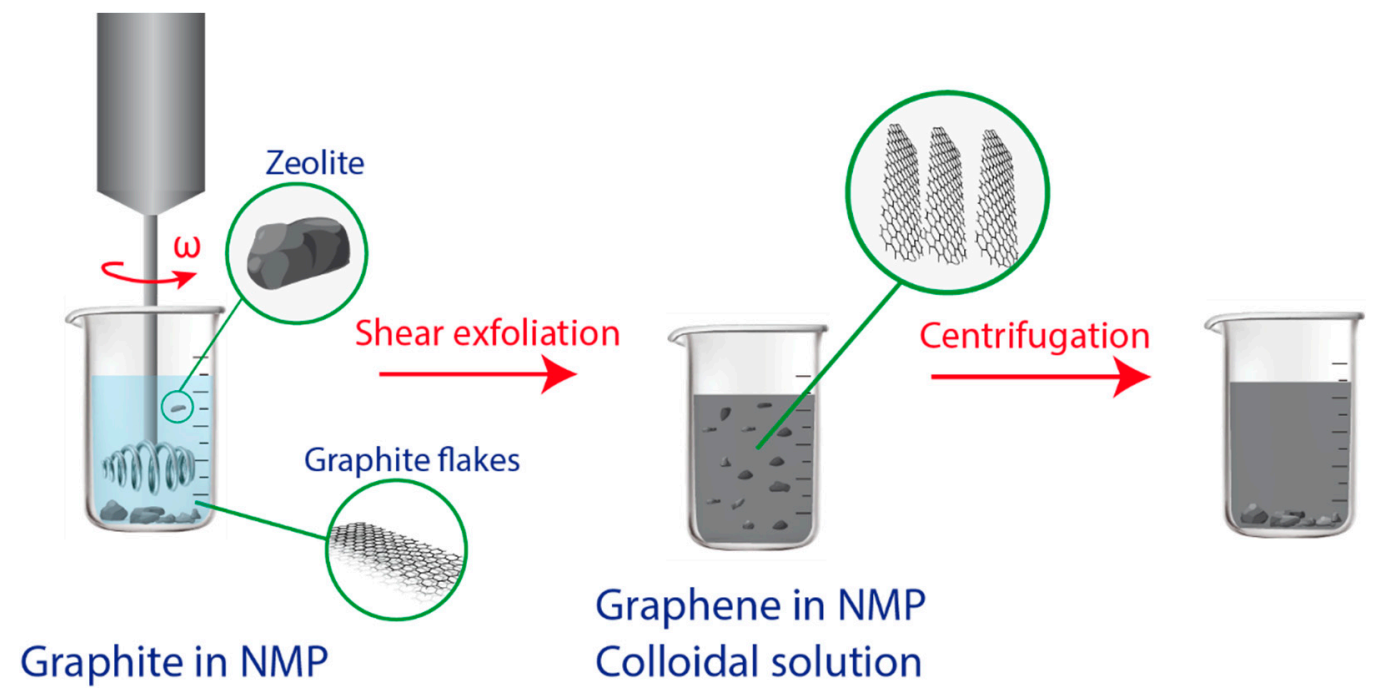

Figure 1. Schematic route of the preparation process for graphene dispersion.

We confirmed that the dispersed material is graphene by performing Raman spectroscopy on deposited films. Raman spectrum of graphite, graphene dispersed in NMP, and graphene dispersed in $\mathrm{NMP}+\mathrm{Z} 4 \mathrm{~A}$ are reported in Figure 2. As is typical for solution-processed graphene, the three main peaks are detected, i.e., the $D$ peak at $\sim 1346 \mathrm{~cm}^{-1}$, the $G$ peak at $\sim 1579 \mathrm{~cm}^{-1}$ and the $2 \mathrm{D}$ peak at $\sim 2719 \mathrm{~cm}^{-1}$. 
The D peak (and the $\mathrm{D}^{\prime}$ peak) is ascribed to structural imperfections while the $\mathrm{G}$ peak is related to the $s p^{2}$-hybridized carbon bonds in the graphene lattice [32]. The peak ratio between the intensity of $\mathrm{D}$ and $G$ peaks $\left(I_{D} / I_{G}\right)$ increases from 0.02 (pristine graphite, Figure $2 c$ ) to 0.10 (graphene dispersed in $\mathrm{NMP}+\mathrm{Z} 4 \mathrm{~A}$, Figure 2a), indicating that very low defects are induced during the shear exfoliation.

The D peak was found here to be narrow and considerably less intense than the $G$ peak. The structure of the D peak is similar to what is usually found for graphene nanosheets [33]. However, the low-intensity ratios, i.e., $I_{D} / I_{G}=0.08$ and $I_{D} / I_{G}=0.10$, are related to the nanosheet edges rather than basal in-plane defects [32,34]. The latter is confirmed because a clear signature of the D' peak (usually associated with $s p^{3}$-type defects and vacancy-type defects [32]) is absent in the Raman spectrum after shear exfoliation (Figure 2a,b). This strongly demonstrates that zeolite-assisted shear exfoliation does not induce edge defects and basal in-plane defects (or impurities adsorbed on the graphene surface [32]). To confirm the absence of small-fragmented zeolite particles into graphene dispersion, Suplementary Figure S1 displays the Raman spectrum of Z4A which is absent in the Raman spectrum obtained by mapping a wide region of graphene sample. This is a crucial result for scaling up for industrial use.

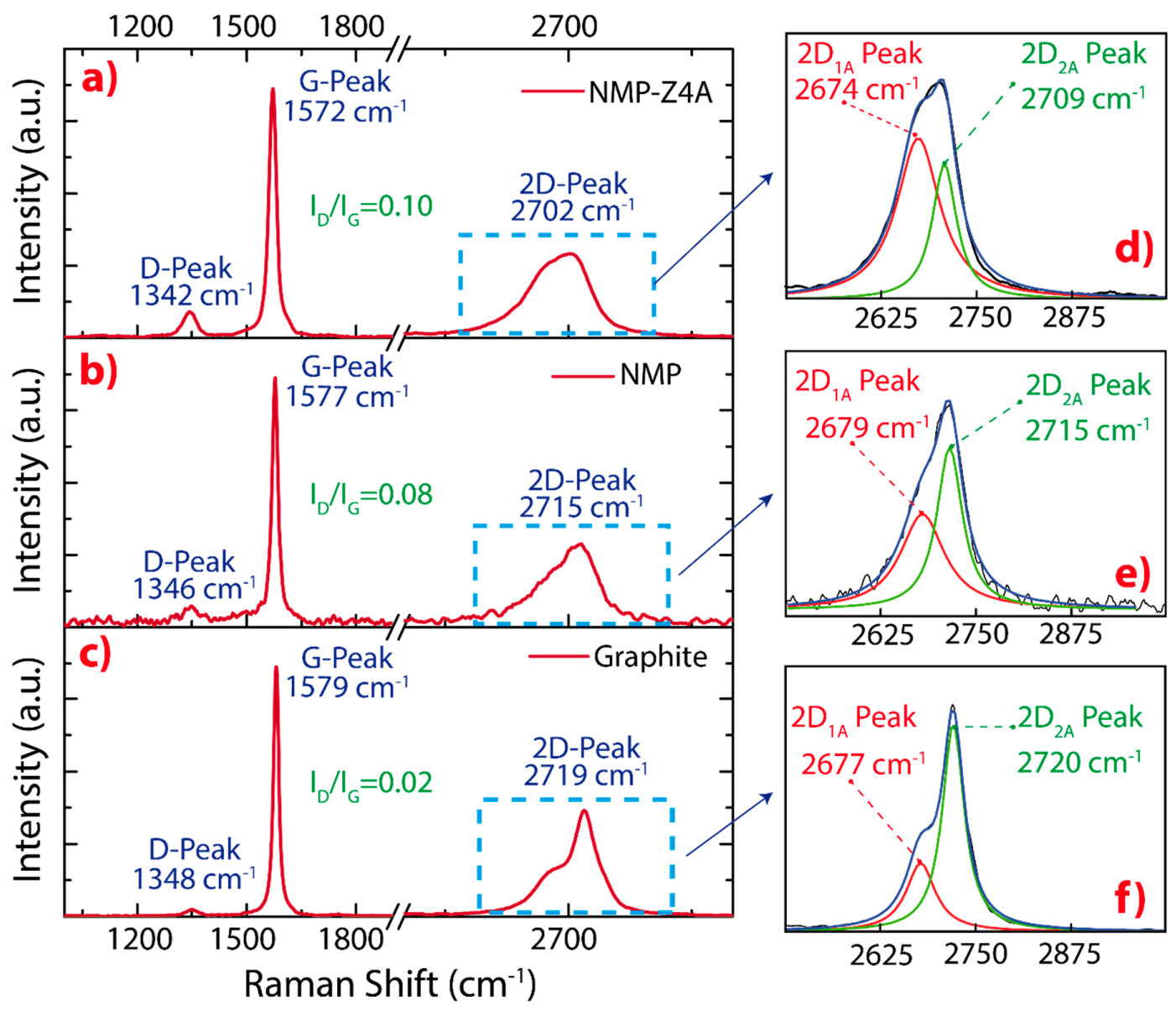

Figure 2. Raman spectrum of (a) graphene dispersed in NMP+Z4A, (b) graphene dispersed in NMP, and (c) pristine graphite. (d-f) Fitting of the 2D peaks with two Lorentzian functions. The intensity normalized by the $\mathrm{G}$ peak.

In Figure 2d, the 2D peak (regularly used to estimate the number of layers in obtained graphene) is reasonably symmetric but less intense than the $G$ peak, indicating the dispersed material in NMP+Z4A is dominated by few-layer graphene (FLG) [35]. To corroborate this fact, we used the full width at half maximum (FWHM) to evaluate the 2D peak. FWHM qualitatively describes the number of layers from single- to five-layer graphene [36]. By fitting the 2D peak with two Lorentzian functions (Figure 2d-f), 
we can note that, the intensity of the 2D1A peak (detected at $\sim 2674 \mathrm{~cm}^{-1}$ ) increases in graphene dispersed in $\mathrm{NMP}+\mathrm{Z} 4 \mathrm{~A}$, with respect to the intensity of the 2D1A peak (detected at $\sim 2677 \mathrm{~cm}^{-1}$ ) in pristine graphite and the $2 \mathrm{D}_{1 \mathrm{~A}}$ peak (detected at $\sim 2679 \mathrm{~cm}^{-1}$ ) in graphene dispersed only in NMP. The latter suggests that $\mathrm{Z} 4 \mathrm{~A}$ improves the shear exfoliation process.

Most importantly, the FWHM values of graphene dispersed in NMP $+Z 4 A$, i.e., $2 \mathrm{D} 1 \mathrm{~A}=69.32 \pm$ 1.24 and $2 \mathrm{D} 2 \mathrm{~A}=38.84 \pm 1.21$, are in good agreement with previous reports for bi- and tri-layer graphene [35,36], confirming the presence of FLG in obtained graphene dispersions, likely, no more than five layers. Interestingly enough, the FWHM values of graphene dispersed only in NMP, i.e., $2 \mathrm{D} 1 \mathrm{~A}=70.51 \pm 3.74$ and $2 \mathrm{D} 2 \mathrm{~A}=40.92 \pm 1.74$, are slightly different compared to respective values of graphene dispersed in NMP+Z4A. This outcome (and the analysis of intensities commented above) supports the hypothesis that zeolite-assisted shear exfoliation is superior to shear exfoliation without the presence of $\mathrm{Z} 4 \mathrm{~A}$.

To further characterize the obtained FLG, we performed microscopic characterization. SEM images are reported in Figure $3 \mathrm{a}, \mathrm{c}$, which correspond to precipitate and supernatant, respectively. Figure 3a shows a non-exfoliated graphite flake covered by zeolite particles, after $6 \mathrm{~h}$ of shear exfoliation and centrifugation. We showed that the Z4A particles are slightly affected at the edges, indicating shear exfoliation does not induce critical structural defects in zeolite cubic morphology. We attributed the edge-fragmented zeolite particles to the collisions between graphite-zeolite and zeolite-zeolite, originated in the dynamic colloidal fluid produced by the mixer. Figure $3 \mathrm{c}$ reveals that the supernatant is composed of smaller and thinner layers.
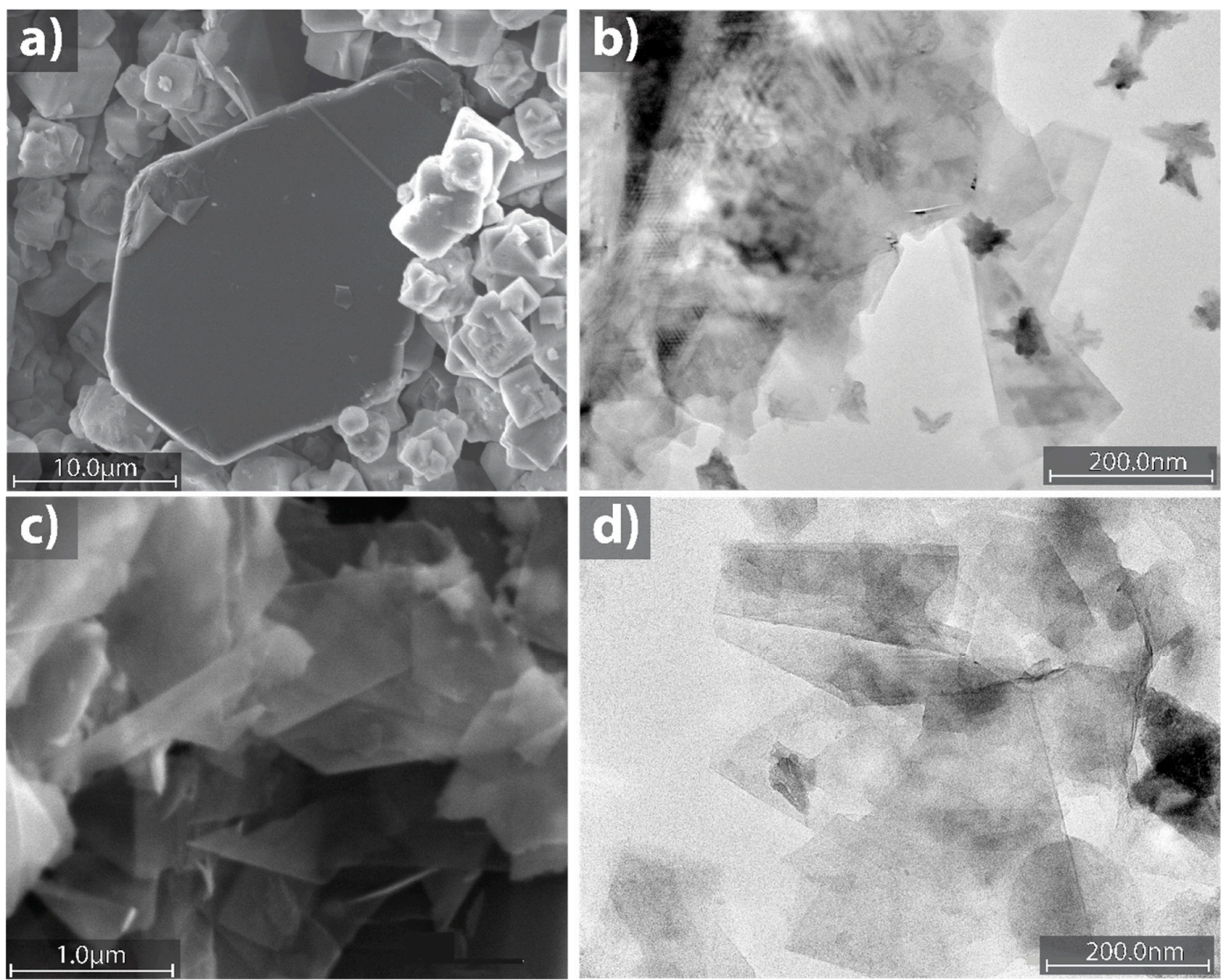

Figure 3. Microscopic analysis after zeolite-assisted shear exfoliation. SEM morphology of (a) non-exfoliated graphite flake interacting with Z4A, recovered as a precipitate and (c) Semitransparent layers obtained from supernatant in NMP+Z4A. TEM image of graphene nanosheets produced (b) by shear exfoliation without $\mathrm{Z} 4 \mathrm{~A}$ and (d) by zeolite-assisted shear exfoliation. 
Similarly to sonication methods [12], we observed small size layers but non-folded edges. Furthermore, these layers seem to be semitransparent and zeolite-free, an important feature for electronic applications. These results suggest that zeolite-assisted shear exfoliation is an excellent alternative in order to preserve the intrinsic properties of the obtained layers.

Thin nanosheets of FLG are reported in Figure 3b,d (TEM images). At first approximation, these nanosheets appear very similar to FLG prepared by sonicating graphite in solvents [12] or aqueous surfactants [29]. Nevertheless, a clear deference is observed between the nanosheets analyzed in this work and those prepared by sonication, i.e., the exfoliated graphene nanosheets by sonication, are mostly folded or folded edge. Figure $3 \mathrm{~d}$ presents well-defined graphene nanosheets obtained by shear exfoliation in NMP+Z4A. On the other hand, Figure $3 b$ shows some dark and distorted regions in graphene nanosheets dispersed only in NMP. The latter result can be interpreted as a poor shear-exfoliation of graphite. This fact was widely discussed and corroborated by Varrla et al. [20] where the authors claim the shear exfoliation can be improved by turbulence, independently of the aqueous media or stabilizing surfactants.

The macroscopic electrical characterization of the obtained FLG on interdigitated gold electrodes is reported in Figure 4. The study is carried out by the 4-wire measurement technique. Four samples were prepared by drop casting of dispersion $(2 \mu \mathrm{L})$ and drying at $100{ }^{\circ} \mathrm{C}$ for $10 \mathrm{~min}$. A relative homogenous dispersion is observed in Figure $4 \mathrm{a}$, this fact is attributed to the small gap between electrodes $(\sim 5 \mu \mathrm{m})$ and the size of the obtained layers around $\sim 1-5 \mu \mathrm{m}$ (Figure 3c). The I-V measurements reported as average values of five measurement cycles and performed on the four prepared samples (Figure $4 \mathrm{~b}$ ), showed a linear flow of current by changing the voltage from $-0.5 \mathrm{~V}$ to $0.5 \mathrm{~V}$. Interestingly enough, we noted good compatibility between the samples, confirming that the samples are covered by homogeneous, well-dispersed and self-interconnected graphene layers. The latter was also demonstrated by extracting the average resistance of each sample, which turned out to be approximately $25 \Omega$ (Figure $4 \mathrm{c}$ ). This simple electrical scrutiny allows us to suggest possible applications in electroanalysis, flow systems and biosensors. Although, drop casting is an appropriate technique for fundamental studies, real applications need an effective adhesion mechanics to deposit the obtained graphene on substrates. However, the adhesive material could affect the electrical properties of graphene. An alternative strategy is reported in Ref [37] by using cyrene and cellulose acetate butyrate (CAB).

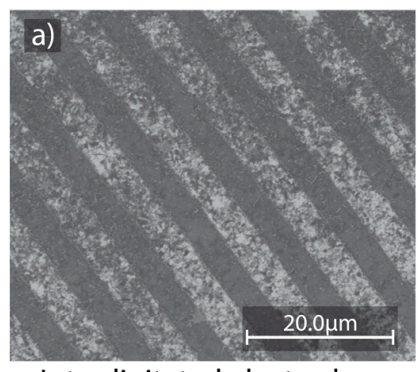

Interdigitated electrodes
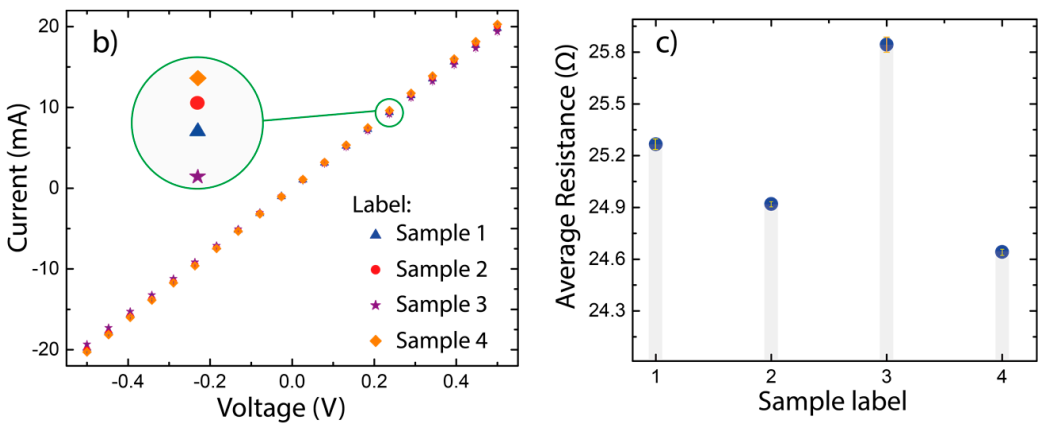

Figure 4. Electrical characterization. (a) SEM image of the obtained FLG dispersion on an interdigitated gold electrode prepared by drop casting of dispersion $(2 \mu \mathrm{L})$. (b) I-V measurements of four prepared samples. (c) Average resistance measured on the four prepared samples. Five measurement cycles are realized at each sample in order to acquire a reliable statistic. The gap between the electrodes is $\sim 5 \mu \mathrm{m}$.

\section{Conclusions}

In conclusion, we have demonstrated that natural graphite flakes can be exfoliated to give defect-free few-layer graphene by a novel zeolite-assisted shear exfoliation method, using a cappuccino mixer to produce shear. The proposed method does not produce acoustic cavitation, high-local shear, and high turbulence; however, the exfoliation of graphite is achieved by moderate turbulence produced by the mixer, which can also be improved by adding the synthetic zeolite $4 \mathrm{~A}$. In comparison to previous 
methods $[19,20]$, we have carried out the exfoliation of graphite in the most basic form of shear exfoliation possible. Most importantly, the obtained layers are free of basal and edge defects which are verified by Raman measurements. Based on the FWHM analysis, we confirmed the presence of few-layer graphene, likely, no more than five layers. SEM and TEM analysis showed semitransparent layers and well-exfoliated graphene nanosheets, respectively. Additionally, the electrical characterization demonstrated that it is possible to prepare graphene-based electrodes with low resistance $(25 \Omega)$. This novel method may be extended to exfoliate inorganic layered materials such as $\mathrm{BN}$ and $\mathrm{MoS}_{2}$.

Supplementary Materials: The following are available online at http://www.mdpi.com/2073-4352/9/8/377/s1, Figure S1: Raman spectrum of (a) zeolite 4A (Z4A) and (b) graphene dispersed in NMP+Z4A after centrifugation for $10 \mathrm{~min}$ at $1000 \mathrm{rpm}$. (Inset) Sampled region.

Author Contributions: All authors contributed to designing and performing measurements, data analysis, scientific discussions, and writing the article.

Funding: This research received no external funding.

Acknowledgments: We acknowledge GraphenTech NL project for the financial support. CVG and GTU wish to thank ITM-CNR for hospitality during the completion of this work.

Conflicts of Interest: The authors declare no conflict of interest.

\section{References}

1. Neto, A.H.C.; Guinea, F.; Peres, N.M.R.; Novoselov, K.S.; Geim, A.K.; Novoselov, K. The electronic properties of graphene. Rev. Mod. Phys. 2009, 81, 109-162. [CrossRef]

2. Coello-Fiallos, D.; Tene, T.; Guayllas, J.; Haro, D.; Haro, A.; Gomez, C.V. DFT comparison of structural and electronic properties of graphene and germanene: Monolayer and bilayer systems. Mater. Today Proc. 2017, 4, 6835-6841. [CrossRef]

3. Sindona, A.; Pisarra, M.; Gomez, C.V.; Riccardi, P.; Falcone, G.; Bellucci, S. Calibration of the fine-structure constant of graphene by time-dependent density-functional theory. Phys. Rev. B 2017, 96, 201408. [CrossRef]

4. Gomez, C.V.; Robalino, E.; Haro, D.; Tene, T.; Escudero, P.; Haro, A.; Orbe, J. Structural and Electronic Properties of Graphene Oxide for Different Degree of Oxidation. Mater. Today: Proc. 2016, 3, 796-802.

5. Soldano, C.; Mahmood, A.; Dujardin, E. Production, properties and potential of graphene. Carbon 2010, 48, 2127-2150. [CrossRef]

6. Yi, M.; Shen, Z. A review on mechanical exfoliation for the scalable production of graphene. J. Mater. Chem. A 2015, 3, 11700-11715. [CrossRef]

7. Gomez, C.V.; Pisarra, M.; Gravina, M.; Riccardi, P.; Sindona, A. Plasmon properties and hybridization effects in silicene. Phys. Rev. B 2017, 95, 085419. [CrossRef]

8. Novoselov, K.; Geim, A.K.; Morozov, S.; Jiang, D.; Zhang, Y.; Dubonos, S.V.; Grigorieva, I.V.; Firsov, A.A. Electric Field Effect in Atomically Thin Carbon Films. Science 2004, 306, 666-669. [CrossRef]

9. Pei, S.; Cheng, H.M. The reduction of graphene oxide. Carbon 2012, 50, 3210-3228. [CrossRef]

10. Usca, G.T.; Gomez, C.V.; Fiallos, D.C.; Tavolaro, P.; Martino, G.; Caputi, L.S.; Tavolaro, A. Preparation of graphene oxide as biomaterials for drug adsorption. AIP Conf. Proc. 2015, 1646, 79-86.

11. Coello Fiallos, D.; Vacacela Gomez, C.; Tubon Usca, G.; Pérez, D.C.; Tavolaro, P.; Martino, G.; Tavolaro, A. Removal of acridine orange from water by graphene oxide. AIP Conf. Proc. 2015, 1646, 38-45.

12. Hernandez, Y.; Nicolosi, V.; Lotya, M.; Blighe, F.M.; Sun, Z.; De, S.; McGovern, I.T.; Holland, B.; Byrne, M.; Gun'Ko, Y.K.; et al. High-yield production of graphene by liquid-phase exfoliation of graphite. Nat. Nanotechnol. 2008, 3, 563-568. [CrossRef] [PubMed]

13. Su, C.Y.; Lu, A.-Y.; Xu, Y.; Chen, F.-R.; Khlobystov, A.N.; Li, L.-J. High-Quality Thin Graphene Films from Fast Electrochemical Exfoliation. ACS Nano 2011, 5, 2332-2339. [CrossRef] [PubMed]

14. Usca, G.T.; Hernandez-Ambato, J.; Pace, C.; Caputi, L.; Tavolaro, A. Liquid-phase exfoliated graphene self-assembled films: Low-frequency noise and thermal-electric characterization. Appl. Surf. Sci. 2016, 380, 268-273. [CrossRef]

15. Gomez, C.V.; Tene, T.; Guevara, M.; Usca, G.T.; Colcha, D.; Brito, H.; Molina, R.; Bellucci, S.; Tavolaro, A. Preparation of Few-Layer Graphene Dispersions from Hydrothermally Expanded Graphite. Appl. Sci. 2019, 9, 2539. [CrossRef] 
16. Ciesielski, A.; Samorì, P. Graphene via sonication assisted liquid-phase exfoliation. Chem. Soc. Rev. 2014, 43, 381-398. [CrossRef] [PubMed]

17. Khan, U.; O'Neill, A.; Lotya, M.; De, S.; Coleman, J.N. High-Concentration Solvent Exfoliation of Graphene. Small 2010, 6, 864-871. [CrossRef] [PubMed]

18. Ou, E.; Xie, Y.; Peng, C.; Peng, H.; Xiong, Y.; Song, Y.; Xu, W. High concentration and stable few-layer graphene dispersions prepared by the exfoliation of graphite in different organic solvents. RSC Adv. 2013, 3, 9490. [CrossRef]

19. Paton, K.R.; Varrla, E.; Backes, C.; Smith, R.J.; Khan, U.; O’Neill, A.; Boland, C.S.; Lotya, M.; Istrate, O.M.; King, P.; et al. Scalable production of large quantities of defect-free few-layer graphene by shear exfoliation in liquids. Nat. Mater. 2014, 13, 624-630. [CrossRef]

20. Varrla, E.; Paton, K.; Backes, C.; Harvey, A.; Smith, R.J.; McCauley, J.; Coleman, J.N. Turbulence-assisted shear exfoliation of graphene using household detergent and a kitchen blender. Nanoscale 2014, 6, 11810-11819. [CrossRef]

21. Guardia, L.; Paredes, J.I.; Rozada, R.; Villar-Rodil, S.; Martínez-Alonso, A.; Tascón, J.M.D. Production of aqueous dispersions of inorganic graphene analogues by exfoliation and stabilization with non-ionic surfactants. RSC Adv. 2014, 4, 14115-14127. [CrossRef]

22. Uddin, M.E.; Kuila, T.; Nayak, G.C.; Kim, N.H.; Ku, B.-C.; Lee, J.H. Effects of various surfactants on the dispersion stability and electrical conductivity of surface modified graphene. J. Alloys Compd. 2013, 562, 134-142. [CrossRef]

23. Zhang, X.; Coleman, A.C.; Katsonis, N.; Browne, W.R.; Van Wees, B.J.; Feringa, B.L. Dispersion of graphene in ethanol using a simple solvent exchange method. Chem. Commun. 2010, 46, 7539. [CrossRef] [PubMed]

24. Schoeman, B.J.; Sterte, J.; Otterstedt, J.E. Colloidal zeolite suspensions. Zeolites 1994, 14, 110-116. [CrossRef]

25. Mintova, S.; Bein, T. Microporous Films Prepared by Spin-Coating Stable Colloidal Suspensions of Zeolites. Adv. Mater. 2001, 13, 1880-1883. [CrossRef]

26. Schoeman, B.J.; Sterte, J.; Otterstedt, J.-E. Synthesis of Colloidal Suspensions of Zeolite ZSM-2. J. Colloid Interface Sci. 1995, 170, 449-456. [CrossRef]

27. Coello-Fiallos, D.; Espin-Lagos, S.M.; Vacacela Gomez, C.; Tavolaro, A.; Caputi, L.S. Comparison of pure membrane of $13 \mathrm{X}$ and 5A zeolite for removal of acridine orange dye from aqueous solutions. Periodico Tche Quimica. 2018, 15, 251-256.

28. Ogilvie, S.P.; Large, M.J.; Fratta, G.; Meloni, M.; Canton-Vitoria, R.; Tagmatarchis, N.; Massuyeau, F.; Ewels, C.P.; King, A.A.K.; Dalton, A.B. Considerations for spectroscopy of liquid-exfoliated 2D materials: Emerging photoluminescence of N-methyl-2-pyrrolidone. Sci. Rep. 2017, 7, 16706. [CrossRef]

29. Lotya, M.; King, P.J.; Khan, U.; De, S.; Coleman, J.N. High-Concentration, Surfactant-Stabilized Graphene Dispersions. ACS Nano 2010, 4, 3155-3162. [CrossRef]

30. Higgins, T.M.; Boland, C.S.; Hanlon, D.; Coleman, J.N.; Backes, C.; Kelly, A.; Harvey, A. Guidelines for Exfoliation, Characterization and Processing of Layered Materials Produced by Liquid Exfoliation. Chem. Mater. 2016, 29, 243-255.

31. Niu, L.; Coleman, J.N.; Zhang, H.; Shin, H.; Chhowalla, M.; Zheng, Z. Production of two-dimensional nanomaterials via liquid-based direct exfoliation. Small 2016, 12, 272-293. [CrossRef] [PubMed]

32. Eckmann, A.; Felten, A.; Mishchenko, A.; Britnell, L.; Krupke, R.; Novoselov, K.S.; Casiraghi, C. Probing the Nature of Defects in Graphene by Raman Spectroscopy. Nano Lett. 2012, 12, 3925-3930. [CrossRef] [PubMed]

33. Pawbake, A.S.; Mishra, K.; Machuno, L.G.; Gelamo, R.V.; Ravindran, T.; Rout, C.S.; Late, D.J. Temperature and pressure dependent Raman spectroscopy of plasma treated multilayer graphene nanosheets. Diam. Relat. Mater. 2018, 84, 146-156. [CrossRef]

34. O'Neill, A.; Khan, U.; Nirmalraj, P.N.; Boland, J.; Coleman, J.N. Graphene Dispersion and Exfoliation in Low Boiling Point Solvents. J. Phys. Chem. C 2011, 115, 5422-5428. [CrossRef]

35. Ferrari, A.C.; Meyer, J.; Scardaci, V.; Casiraghi, C.; Lazzeri, M.; Mauri, F.; Piscanec, S.; Jiang, D.; Novoselov, K.; Roth, S.; et al. Raman Spectrum of Graphene and Graphene Layers. Phys. Rev. Lett. 2006, 97, 187401. [CrossRef] [PubMed] 
36. Hao, Y.; Wang, Y.; Wang, L.; Ni, Z.; Wang, Z.; Wang, R.; Koo, C.K.; Shen, Z.; Thong, J.T.L. Probing Layer Number and Stacking Order of Few-Layer Graphene by Raman Spectroscopy. Small 2010, 6, 195-200. [CrossRef]

37. Pan, K.; Fan, Y.; Leng, T.; Li, J.; Xin, Z.; Zhang, J.; Hao, L.; Gallop, J.; Novoselov, K.S.; Hu, Z. Sustainable production of highly conductive multilayer graphene ink for wireless connectivity and IoT applications. Nat. Commun. 2018, 9, 5197. [CrossRef] [PubMed]

(C) 2019 by the authors. Licensee MDPI, Basel, Switzerland. This article is an open access article distributed under the terms and conditions of the Creative Commons Attribution (CC BY) license (http://creativecommons.org/licenses/by/4.0/). 